\title{
AEROSOL INFLUENCE ON PHOTOVOLTAIC SOLAR ENERGY GENERATION FOR THE STATE OF GOIÁS (BRAZIL)
}

\author{
C. R. Silveira Júnior ${ }^{1 *}$, G. C. Luiz ${ }^{2}$, G. A. S. Barbosa ${ }^{1}$, L. G. C. Sousa ${ }^{1}$ \\ ${ }^{1}$ Federal Institute of Education, Science and Technology of Goiás - IFG \\ Department IV, Research Lab \\ carlos.junior@ifg.edu.br,(gustavo.souteras, cardoso.gabriel)@academico.ifg.edu.br \\ ${ }^{2}$ Federal University of Goiás, Institute of Socio-Environmental Studies - IESA \\ gislaineluiz@yahoo.com.br
}

KEY WORDS: Aerosols, energy generation, GOES-16, solar photovoltaic, air pollution

\begin{abstract}
:
The presence of aerosols in atmosphere has the potential to decrease the efficiency of solar photovoltaic energy generation. The central region of Brazil presents a remarkable growth of these aerosols between March and October, when the region undergoes a period of intensive slash and burn agriculture. This paper aims to evaluate the impact of this anthropogenic aerosol on electric power generation by comparing data from geostationary satellites with photovoltaic power generation data.
\end{abstract}

\section{INTRODUCTION}

Natural resources shortage and particularly climate change have caused in recent years several changes in the relationship between society and environment. Those changes include new forms of energy generation, such as photovoltaic solar energy generation. One of the largest countries in the world with radiation levels to generate photovoltaic energy, Brazil boosted its economic efficiency. According to Brazilian Association of Photovoltaic Solar Energy (Absolar), the price of photovoltaic modules has fallen by around $80 \%$ over the last ten years, completing in 2019 the 1st Gigawatt of installed generation PV capacity. (ABSOLAR, 2019)

However, anthropogenic actions and climate change directly influence photovoltaic solar energy generation. Studies conducted by Calinoiu (2013) show a drop of $20 \%$ in the efficiency of energy generation in the presence of aerosols in the atmosphere. This fact is of particular concern in places such as the central region of Brazil, which has high solar insolation that enables the generation of electricity by photovoltaic panels, but lose effectiveness due to the increased agricultural burning between March and August (INPE, 2019). Therefore, it is important to carry out studies in order to identify this influence from aerosol data.

Aerosol data can be obtained from surface stations, such as AERONET (AErosol RObotic NETwork), but have a sparse distribution with low temporal resolution and low spatial resolution $\left(0.5 \times 0.5^{\circ}\right)$ (Garay et al., 2019). On the other hand, geostationary orbiting satellite images from GOES-16 and GOES-17 (Geostationary Operational Environmental Satellites) satellites continuously scan the same region of the globe, in the same position, with a temporal resolution of 15 minutes and spatial resolution of $2 \mathrm{~km}$ (GOES, 2019).

This paper aims at evaluating the relationship between the aerosol product generated by the GOES satellite and the generation of electricity by photovoltaic panels.

\footnotetext{
*Corresponding author
}

\section{GOES SERIES: AEROSOL PRODUCT}

GOES-16 is part of the Geostationary Operational Environmental Satellites (GOES) project, a series of geostationary satellites developed for climatological purposes. The geostationary nature provides the ability to perform continuous scans of the same region of the globe at the same position at shorter time intervals compared to polar orbit systems. GOES-16 is positioned at $75.2^{\circ} \mathrm{W}$, on the Equator line, covering eastern North America and all of South America.

The GOES-16 satellite has several sensors on board, among them, the Advanced Baseline Imager (ABI), which has a spatial resolution of 500,1,000 and 2,000 meters, depending on the spectral band, and 16 bands between 0.47 and $13.30 \mu \mathrm{m}$. This sensor has three forms of imaging being American continent images (Full Disk) every 15 minutes, Pacific US images every 5 minutes, and images every 30 seconds from a small portion of the west coast of the United States.

Remote sensing allows, from radiance or irradiance values integrated into certain spectral bands, to obtain the physical quantities related to the object with which radiance interacted (King et al., 1978). To estimate aerosol over continent, the bands $0.47,0.64 \mu$ (visible), 0.86, 1.37 and $2.20 \mu \mathrm{m}$ (near infrared) are used (GOES, 2019).

GOES products are available for download from various servers such as Amazon's AWS (Open Commons Consortium, 2019) in Network Common Data Form (NetCDF) format. Each file represents the result of a Full Disk, CONUS, or Mesoscale scan. These products are identified by acronyms and basically divided into two levels.

Several factors can interfere with the reading made by the sensors, such as the presence of clouds. Based on the calculation of the interference of each of these factors in the value of the pixel read, it is associated to each pixel a value that signals the quality of this reading, the Quality Flag, which has value 0 (zero) to 3 (three) given at integer intervals whose value signals the highest level of confidence in the AOD value presented (GOES, 2019). 
Thus, the AOD (Aerosol Optical Depth) product consists of an image with pixel values identifying the extent of extinction due to atmospheric aerosols at a wavelength of $550 \mathrm{~nm}$ along with the quality bit, and these values are a dimensionless quantity. Its spatial resolution is $2 \mathrm{~km}$, its temporal resolution is 15 minutes.

For the purposes of this study, AOD values that presented Quality Flag greater than one were excluded. The use of pixels that signaled medium or high quality was a choice made in order to improve the effective error resulting from the statistical analysis of these data.

\section{STUDIED AREA}

The State of Goiás is located in the Midwest region of Brazil, within the Cerrado biome, having only a small part southeast of Goiás State characterized as Atlantic Forest. The state capital of Goiás is the city of Goiânia which is located on an imaginary dividing line between the tropical and the subtropical zone. By analyzing the climatological data of the region, it has proven difficult to fit it between the two classic divisions of warm climates, i.e. hot and humid or hot and dry (Fernandes, 2002).

Generally speaking, the region where Goiânia is located has two significant characteristics: continentality, because it is very far from the Atlantic coast and the great regularity of the cyclic process of air mass displacement, which combined with other factors, presents a rainy season (having five months with rainfall around $200 \mathrm{~mm}$ per month) and a drought season (with two months of light rain and three months of drought). Besides that, it has a wind regime of evident direction (Fernandes, 2002).

To carry out the research, it was delimited a sector associated to the location of IFG (Federal Institute of Education, Science and Technology of Goiás), Campus Goiânia in order to have better control of data processing. IFG has a solar photovoltaic pilot plant installed on its roof, on NExT (Alternative Energy Sources Laboratory of the Experimental and Technological Studies Center) as can be viewed in Figure 1.

The photovoltaic pilot plant has 6 panels connected in series totaling $240 \mathrm{~W}$ of power, connected to a $2,000 \mathrm{~W}$ single phase inverter. The site has incidence of solar radiation throughout the day. They were mounted with a slope of $17^{\circ}$ (to the latitude of the city of Goiannia), in the direction of $18^{\circ}$ west of the geographic north, following the direction of the roof. The plant has sensors that provide indicators of electric power generation, being electric voltage, electric current, electric power and ambient temperature. The sample interval was from March to May 2019, the final period of the rainy season in Goiás State with little cloud.

\section{METHODOLOGY}

For processing GOES satellite data, an algorithm was developed to convert aerosol product data into georeferenced images. For this, the Python programming language with GDAL library (Open Source Geospatial Foundation, 2019) were used. Python routines were developed for aerosol product processing in order to generate maps, graphs and analysis of aerosol distribution in the atmosphere. In addition to the aerosol optical depth values, it also generates a georeferenced image of the quality bits present in the AOD product, the Quality Flag used in filtering samples to maintain data processing reliability. To

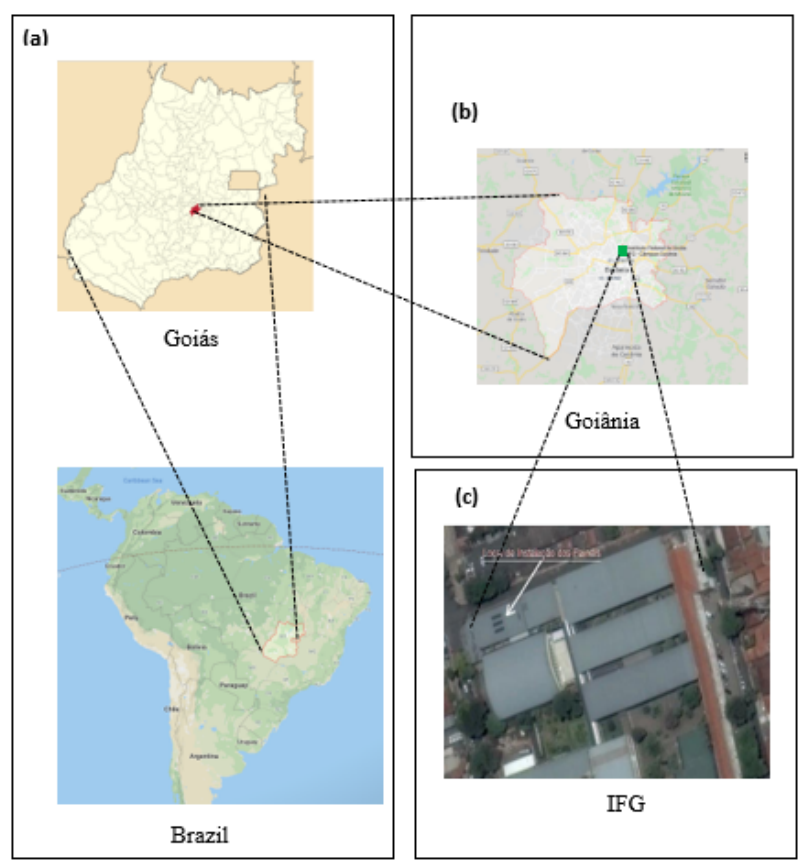

Figure 1. (a) Location of Goiás State within the Brazilian territory, (b) Location of IFG within the city of Goiânia. (c) Solar plant used for data acquisition.

validate the data processing scripts, the results were compared with the images processed by the QGIS software (Quantum GIS).

Data from the solar photovoltaic plant acquisition system were averaged every 5 minutes, every 10 minutes and every 15 minutes to facilitate comparison between such data and data from the GOES satellite. QGIS is an open source desktop and multiplatform geographic information system application designed to allow the user to perform visualization, editing and analysis of geospatial data, and is widely used in remote sensing data processing (QGIS, 2019).

With the processed data, the relationship between AOD and the power generation indicators such as electric voltage, electric current and electric power, besides the ambient temperature, was evaluated. Daytime sample charts and statistical correlation graphs between AOD and power generation indicators were created.

To analyze the difference in photovoltaic solar energy generation, AOD samples were grouped into two groups: values less than 0.1 and values between 0.1 and 0.2 . For this purpose, statistical tests were conducted to evaluate the sample differences of the two groups.

For the analysis aerosol data with Quality Flag equal to 0 (high quality) or 1 (medium quality) was used. Only samples from 8:00 to 18:00 were considered, eliminating noises from the aerosol product at sunrise and sunset intervals. Only the 5-minute average photovoltaic solar energy generation indicators were considered, as they showed a higher statistical correlation with the AOD product.

\section{RESULTS}

Figure 2 presents the aerosol product over Goiás State at dawn. In the west we observe the areas where aerosol readings were 
not possible yet due to absence of solar light (in white and red color). Figure 3 shows the aerosol product over Goiás State after dawn. The influence of clouds to the southeast of Goiás State (in white) and some pixels with a high level of aerosol to the southwest (in red tones) is probably due to agricultural burning.

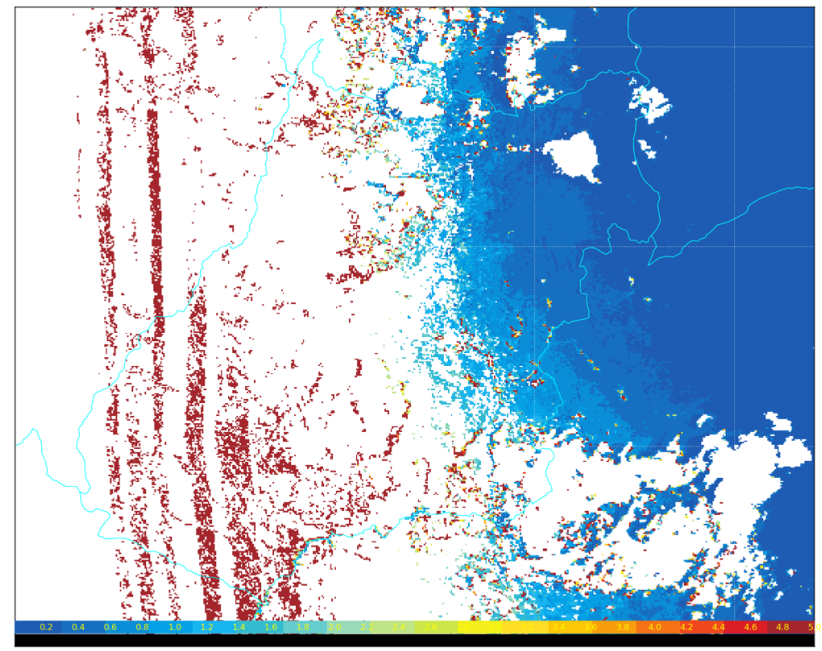

Figure 2. Image generated from aerosol readings for March 31, 2019 at 6:45 am (local time)

The graphs of some parameters' behavior over time are presented in Figure 4, where the graph for voltage and electric current is presented left and the graph for electric power, temperature and AOD is presented right. It can be observed that the graph of the electric voltage presents a behavior apparently similar to the variation of the aerosol, with the variables directly proportional, increasing the electric voltage as the value of AOD increases. On the other hand, the electric current presents a quadratic function behavior with downward concavity, presenting higher values from 12:10 to $13: 40$, the period of greatest solar insolation. This behavior is apparently related to the variation of the solar azimuth angle during the day. It can be also observed by checking the electric power that it presents an inversely proportional relation to the aerosol, presenting higher electric power when the aerosol value is low and lower electric power when the aerosol value is higher. Same behavior were observed in China (Li et al., 2017).

Figure 5(b) presents the correlation graphs between the AOD product and the power generation indicators voltage, electric current, electrical power and ambient temperature. The AOD product has a weak correlation with the indicators of electricity generation, being positive for the voltage $(r=0.33, p<0.05)$ and negative for the electric current. The AOD product has no statistical correlation with temperature $(\mathrm{p}=0.39)$. It should be noted that there was little variation in the aerosol value over the study time interval, considering only data with high and medium quality Quality Flag, where values were only within the range of -0.05 to 0.5 . In fact, a larger sample time interval could provide a greater correlation between aerosol and power generation indicators.

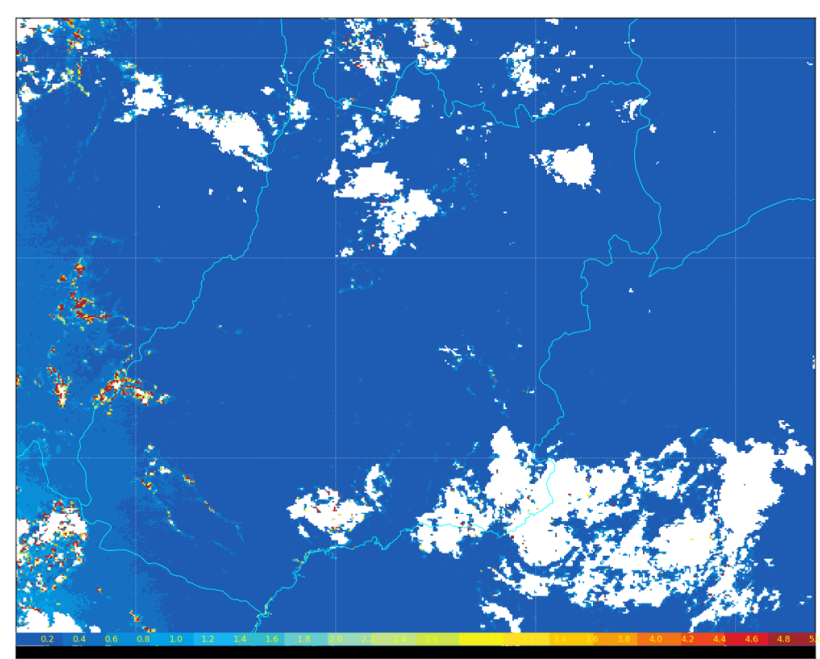

Figure 3. Image generated from aerosol readings for March 31, 2019 at 8:15 am (local time)

In a t-test statistical analysis of independent group, considering the low aerosol groups $(A O D<0.1)$ with 85 samples and the highest aerosol level $(A O D \geq 0.1)$ with 23 samples, Box \& Whisker graphs were generated to illustrate the sample distribution of the two aerosol groups as shown in Figure 6. Higher values of electric voltage are observed as aerosol increases, as well as higher values of electric current and electric power, as aerosol decreases.

The generated voltage averages $178.16 \mathrm{~V}(\sigma=6.82)$ for low aerosol level and $182.56 \mathrm{~V}(\sigma=6.89)$ for higher aerosol level $(F=1.01, d f=106, p<0.05)$. On the other hand, the average current generated was $6.75 \mathrm{~A}(\sigma=1.81)$ for low aerosol level and 5.31 A $(\sigma=2.46)$ for higher aerosol level $(F=1.84$, $d f=106, p<0.05)$. Indeed, the average power of $39.36 \mathrm{~W}$ $(\sigma=5.14)$ for low aerosol level and $37.52 \mathrm{~W}(\sigma=5.48)$ for higher aerosol level $(F=1.13, d f=106, p<0.05)$. The average low aerosol temperature was $38.48^{\circ} \mathrm{C}(\sigma=4.93)$ and the highest aerosol level was $37.52{ }^{\circ} \mathrm{C}(\sigma=5.94)$, not presenting statistical difference between the groups $(F=1.45, d f=106$, $p=0.23$ ). The results confirm that even in a short time observation it is possible to identify differences in the photovoltaic solar energy generation of solar panels according to aerosol variation. 


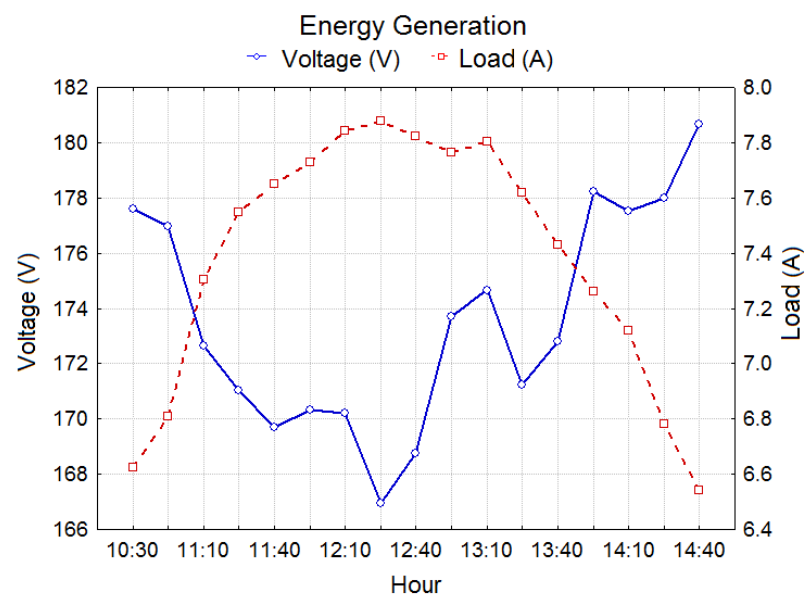

(a) Voltage and load behavior over time.

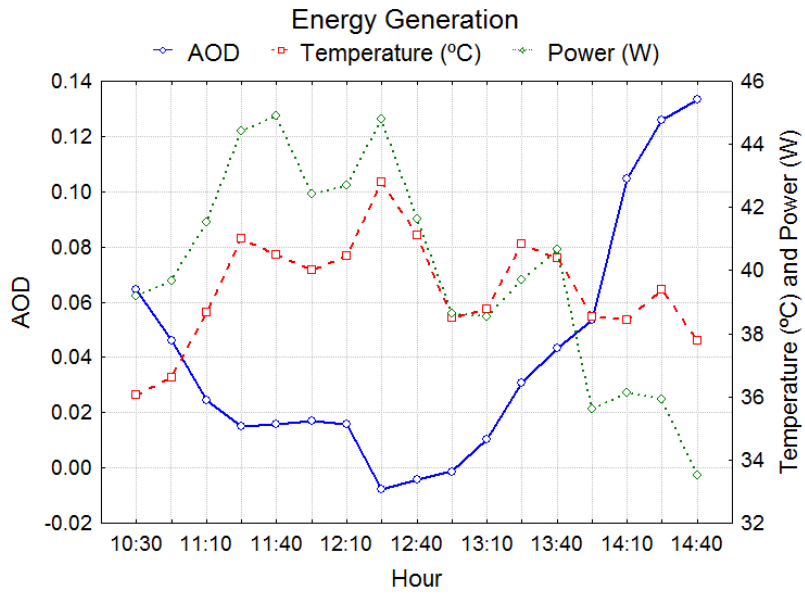

(b) AOD, temperature and power behavior over time.

Figure 4. Some parameters' behavior graphs over time

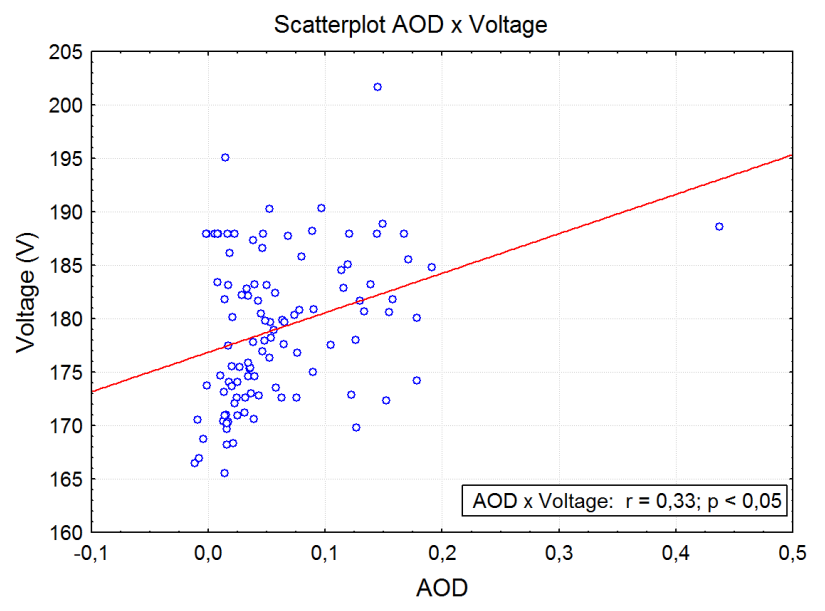

(a) Voltage

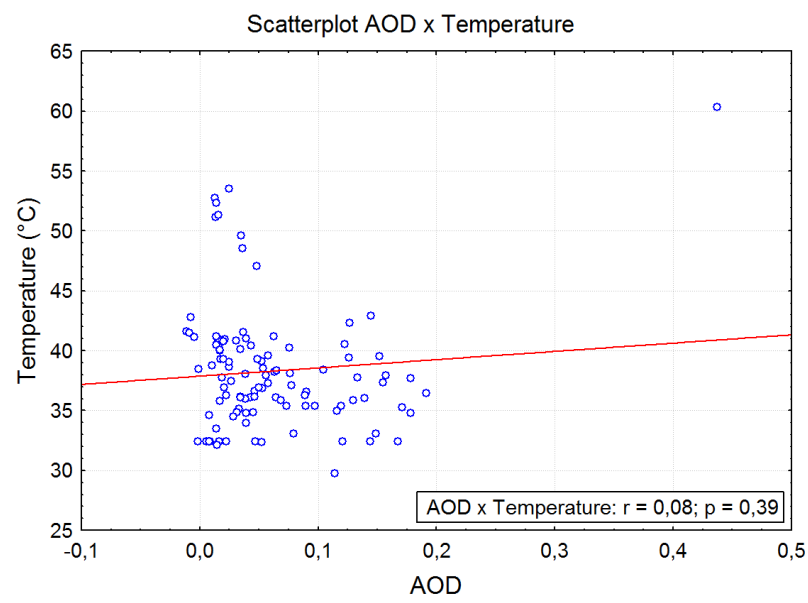

(c) Temperature

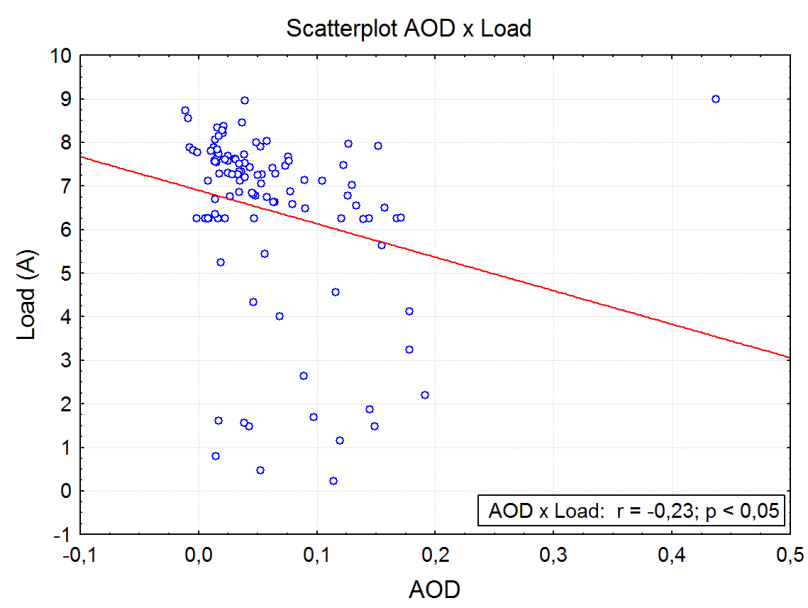

(b) Load

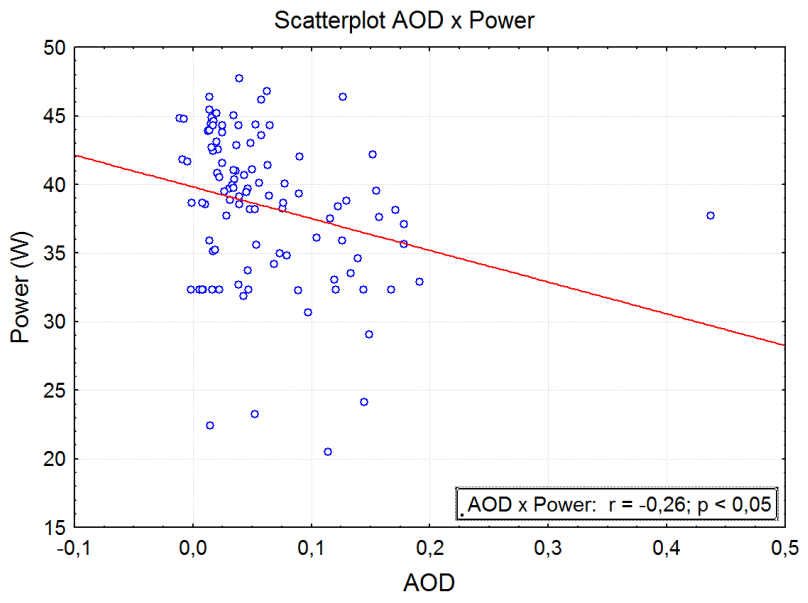

(d) Power

Figure 5. Power generation indicators correlation with AOD 


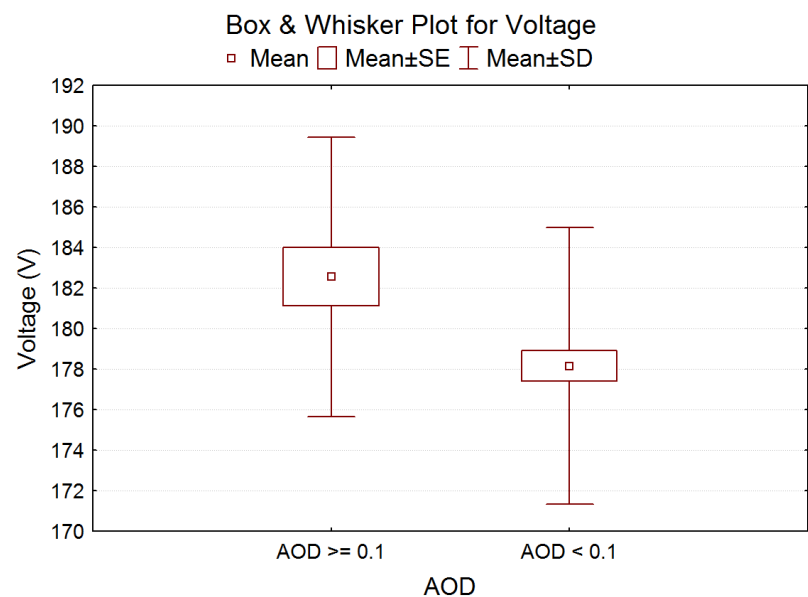

(a) Voltage

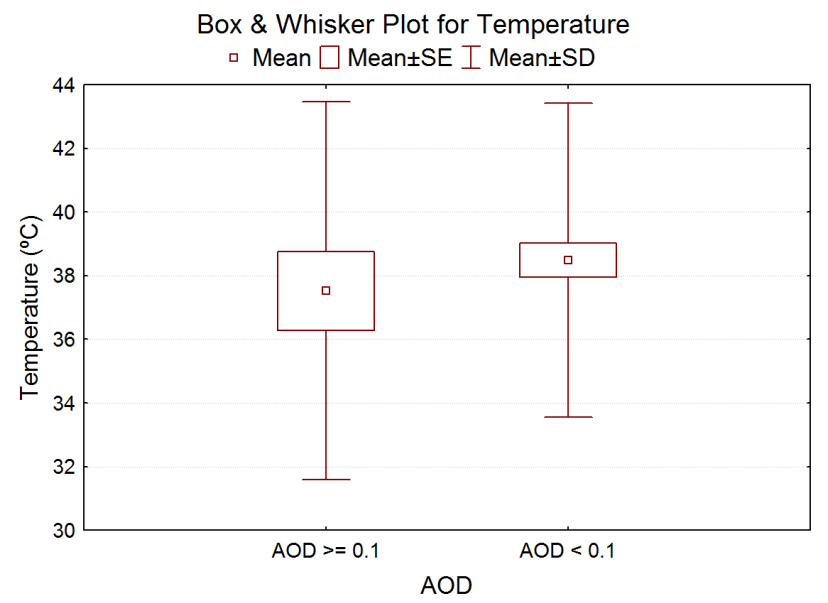

(c) Temperature

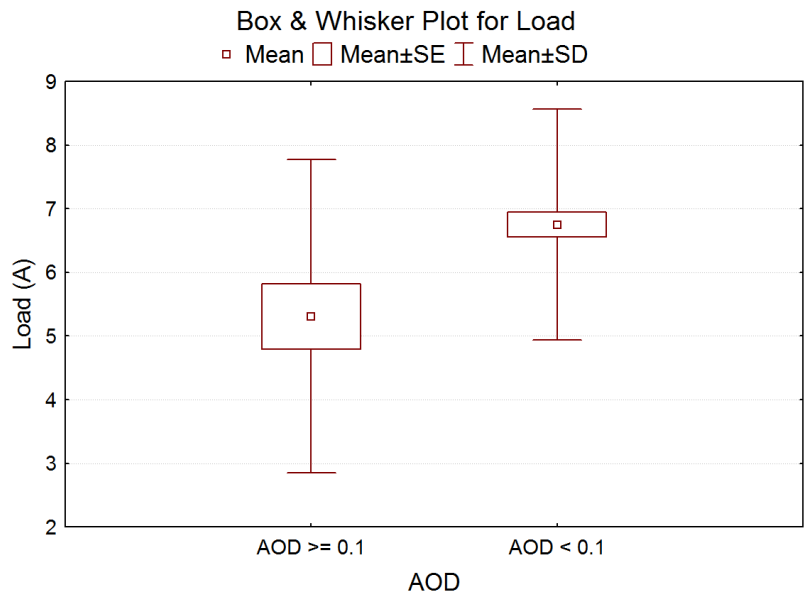

(b) Load

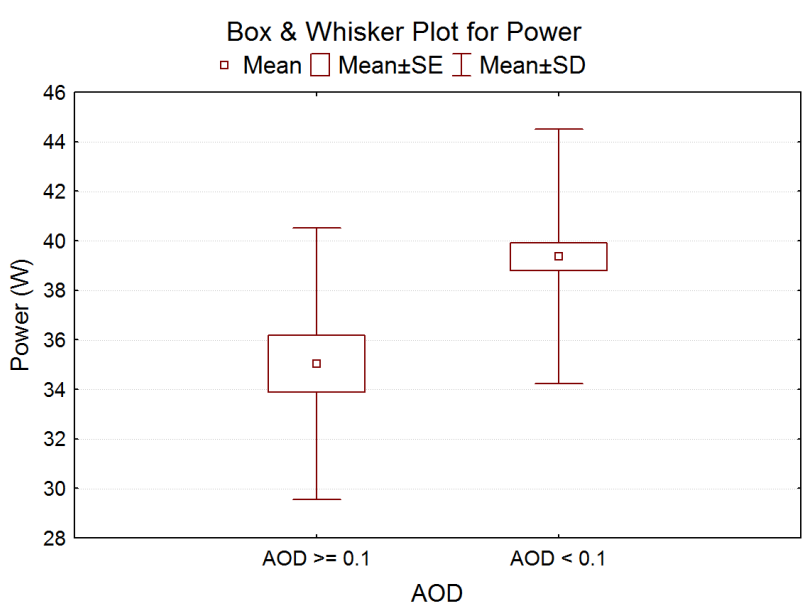

(d) Power

Figure 6. Box \& whisker plot of power generation indicators and AOD

\section{CONCLUSION}

The present study evaluated data from the GOES-16 geostationary satellite with photovoltaic solar energy generation data. The results confirm the correlation between aerosol and photovoltaic solar energy generation indicators. Regarding the aerosol product, positive correlations for electric voltage and negative correlations for current and electric power were observed. However, a larger evaluation interval with quality estimates that could present greater variation in values of the aerosol product would allow a deeper study on this relationship.

Future studies are dedicated to the analysis of a database of longer time interval as well as using data from the photovoltaic solar energy plant, in the final phase of installation, with 775 solar panels totaling $250 \mathrm{kWp}$.

\section{ACKNOWLEDGEMENTS}

We thank CNPq (National Council for Scientific and Technological Development) for the resource available from Call MCTIC/CNPq No 28/2018 - Universal. We thank IFG for their support in developing the project.

\section{References}

ABSOLAR (Associacaoo Brasileira de Energia Solar Fotovoltaica), 2019. Energia solar fotovoltaica atinge primeiro gigawatt no brasil. http://absolar.org.br/energia-solarfotovoltaica-atinge-primeiro-gigawatt-no-brasil.html.

Calinoiu, D., 2013. Influence of aerosols pollution on the amount of collectable solar energy. Energy Conversion and Management, 70.

Fernandes, A. M. C. P., 2002. Clima da Região de Goiânia. Universidade Católica de Goiás - Departamento de Artes e Arquitetura.

Garay, M. J., Witek, M. L., Kahn, R. A., Seidel, F. C., Limbacher, J. A., Bull, M. A., Diner, D. J., Hansen, E. G., Kalashnikova, O. V., Lee, H., Nastan, A. M., Yu, Y., 2019. Introducing the $4.4 \mathrm{~km}$ Spatial Resolution MISR Aerosol Product. Atmospheric Measurement Techniques Discussions, 2019, 1-58. https://www.atmos-meas-tech-discuss.net/amt2019-340/.

GOES-R/Code 416, 2019. Product definition and user's guide (PUG) Volume 5: Level 2+ Products. DCN 7035538. https://www.goes-r.gov/products/docs/PUG-L2+-vol5.pdf. 
INPE (Instituto Nacional de Pesquisas Espaciais), 2019. Programa queimadas. http://queimadas.dgi.inpe.br/queimadas/portal.

King, M., Byrne, D., Herman, B., Reagan, J., 1978. Aerosol size distribution obtained by inversion of optical depth measurements. J. Atmos. Sci, 35.

Li, X., Wagner, F., Peng, W., Yang, J., Mauzerall, D. L., 2017. Reduction of solar photovoltaic resources due to air pollution in China. Proceedings of the $\mathrm{Na}$ tional Academy of Sciences, 114(45), 11867-11872. https://www.pnas.org/content/114/45/11867.

Open Commons Consortium, 2019. Get Data. DCN 7035538. http://edc.occ-data.org/goes16/getdata/.

Open Source Geospatial Foundation, 2019. GDAL documentation . https://gdal.org/.

QGIS, 2019. Guia do Usuário QGIS. https://docs.qgis.org/3.4/pt_BR/docs/user_manual/.

Revised november 2019 